\title{
Disulfide pairings and secondary structure of porcine $\beta$-microseminoprotein
}

\author{
Iren Wang ${ }^{\mathrm{a}, \mathrm{b}}$, Tsun-Ai $\mathrm{Yu}^{\mathrm{a}}$, Shih-Hsiung $\mathrm{Wu}^{\mathrm{b}, \mathrm{c}, *}$, Wen-Chang Chang ${ }^{\mathrm{b}}$, Chinpan Chen ${ }^{\mathrm{a}, * *}$ \\ anstitute of Biomedical Sciences, Academia Sinica, 128 Academia Road, Section 2, Nankang, Taipei 115, Taiwan \\ ${ }^{\mathrm{b}}$ Institute of Biochemical Sciences, National Taiwan University, Taipei 106, Taiwan \\ ${ }^{\mathrm{c}}$ Institute of Biological Chemistry, Academia Sinica, Taipei 115, Taiwan
}

Received 8 January 2003; revised 28 February 2003; accepted 3 March 2003

First published online 1 April 2003

Edited by Thomas L. James

\begin{abstract}
A sperm motility inhibitor isolated from porcine seminal plasma is identical to porcine $\beta$-microseminoprotein (MSP). Circular dichroism (CD) and nuclear magnetic resonance (NMR) data showed that the native and recombinant porcine MSPs exhibit very similar structure. The five disulfide pairings on porcine MSP were unambiguously assigned based on NMR data and further confirmed using structural calculations. Surprisingly, our derived pairings differ from those recently reported for ostrich MSP based on matrix-assisted laser desorption ionization-time of flight (MALDI-TOF) analysis. Furthermore, the secondary structure was determined to comprise one four-stranded and two double-stranded antiparallel $\beta$-sheets. As we know, this is the first detailed secondary structure reported among several types of MSPs.
\end{abstract}

(C) 2003 Published by Elsevier Science B.V. on behalf of the Federation of European Biochemical Societies.

Key words: $\beta$-Microseminoprotein;

Human prostate secretory protein of 94 amino acids; $\mathrm{Na}^{+}, \mathrm{K}^{+}$-adenosine triphosphatase inhibitor;

Nuclear magnetic resonance; Structure

\section{Introduction}

$\beta$-Microseminoprotein (MSP) was originally isolated from human seminal plasma [1], and the human MSP has also been designated as prostate secretory protein of 94 amino acids $\left(\mathrm{PSP}_{94}\right)$ to reveal both the size of the secreted protein and the high concentration in prostatic secretion [2]. A variety of MSPs as well as their primary sequences have subsequently been identified from several different species, including baboon [3], rhesus [4], tamarin [2], pig [5], rat [6], mouse [7] and ostrich [8]. It has been found that MSPs are all non-glycosylated and disulfide bond rich, but show a relatively low level of conservation. This finding suggests that MSPs have undergone a rapid and unusual evolution. Although the putative biological roles, such as immunoglobulin binding factor

\footnotetext{
${ }^{*}$ Corresponding author. Fax: (886)-2-2653 9142.

**Corresponding author. Fax: (886)-2-2788 7641

E-mail addresses: shwu@gate.sinica.edu.tw (S.-H. Wu),

bmchinp@ccvax.sinica.edu.tw (C. Chen).
}

Abbreviations: MSP, $\beta$-microseminoprotein; MALDI-TOF, matrixassisted laser desorption ionization-time of flight; CD, circular dichroism; NOE, nuclear Overhauser enhancement; NOESY, nuclear Overhauser enhancement spectroscopy; TOCSY, total correlation spectroscopy
[9] and tumor marker for gastric carcinoid disease [10], have been suggested for some MSPs, the biological functions that MSPs possess are still poorly understood. In addition, their sequences bear no significant resemblance to proteins of known function, making it difficult to speculate about their biological functions. Therefore, to gain insight into the structure/function relationship of MSPs, a detailed structural analysis of MSPs is necessary. To date, structural studies on MSPs are scarce and there is no known structure related to MSP in the Protein Data Bank. Most recently, three of the five disulfide pairings of ostrich MSP have been identified based on the technique of matrix-assisted laser desorption ionization-time of flight (MALDI-TOF) mass spectrometry [8], but the linkages between $\mathrm{Cys}^{37}$ and $\mathrm{Cys}^{39}$ to the Cys residues composing the pair 46-47 in ostrich MSP however could not be unambiguously assigned.

A sperm motility inhibitor, isolated from porcine seminal plasma, has been confirmed to be identical to porcine MSP based on peptide sequence, amino acid composition and mass spectral analysis [11]. Porcine MSP contains 91 amino acid residues with a pyroglutamate at its $\mathrm{N}$-terminus and is linked with five disulfide bridges. The previous biochemical studies showed that porcine MSP not only inhibits competitively the activity of $\mathrm{Na}^{+}, \mathrm{K}^{+}$-adenosine triphosphatase (ATPase) but also binds to both the head and tail regions of sperm [11]. Multiple sequence alignment of porcine MSP with other MSPs (Fig. 1) shows that all Cys residues are conserved and located at the same positions. One may suggest that the disulfide pairings would be the same based on the conservation of Cys residues. In this work, we cloned, expressed, and purified recombinant unlabeled as well as ${ }^{15} \mathrm{~N} /{ }^{13} \mathrm{C}$ double-labeled porcine MSP to carry out its structural analysis. On the basis of heteronuclear multidimensional nuclear magnetic resonance (NMR) techniques using recombinant ${ }^{15} \mathrm{~N} /{ }^{13} \mathrm{C}$-labeled protein, we identified the five disulfide bond pairings of porcine MSP and surprisingly these disulfide patterns are different from those of ostrich MSP. The detailed secondary structure of porcine MSP was also determined and will be discussed.

\section{Materials and methods}

\subsection{Preparation of porcine MSP}

The native porcine MSP was obtained as previously described [11]. The unlabeled and uniformly ${ }^{15} \mathrm{~N} /{ }^{13} \mathrm{C}$ double-labeled porcine MSP with an additional 17 amino acids, MRGSHHHHHHGSDDDDK, at the N-terminus was expressed in Escherichia coli M15 containing the constructed vector [pQE30] at $37^{\circ} \mathrm{C}$. Isopropyl- $\beta$-thiogalactopyranoside (IPTG) was added to a final concentration of $2 \mathrm{mM}$ when 

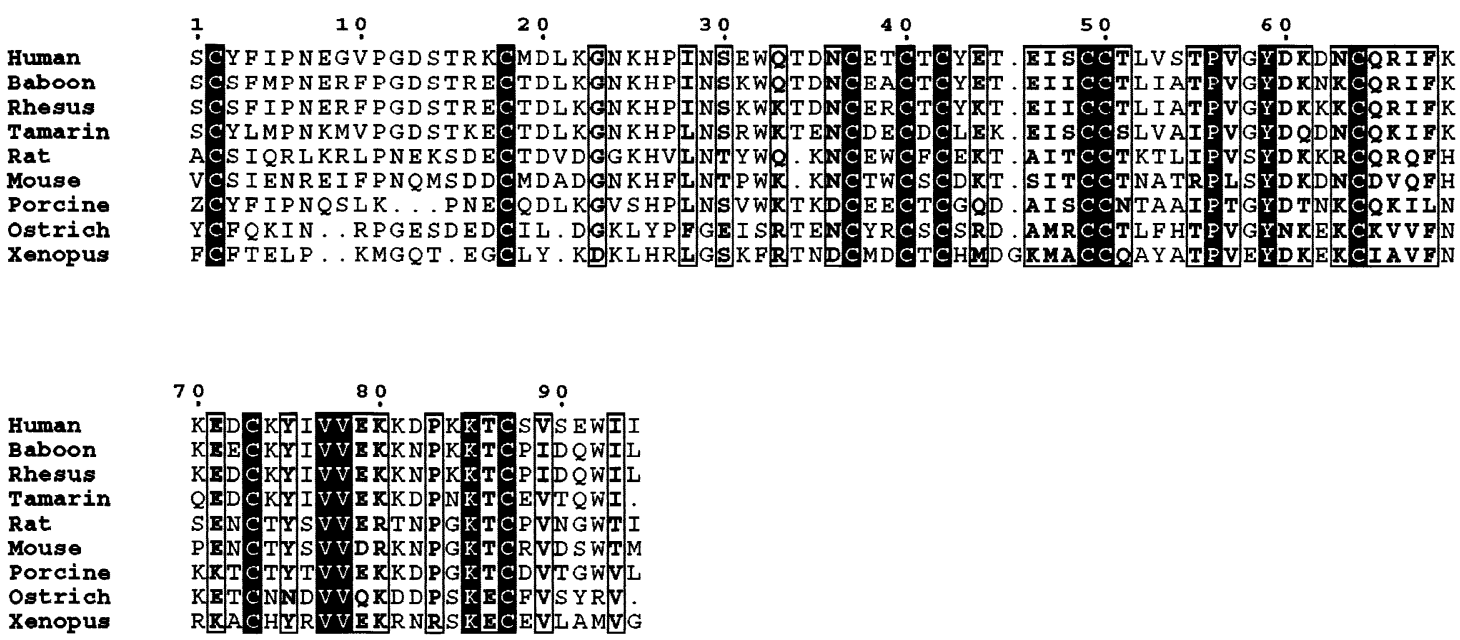

Fig. 1. Multiple sequence alignment of porcine MSP with eight other MSPs was generated using CLUSTAL-W [24] and ESPript [25] programs. Residues that are identical are shown in white type with a black background and those that are highly conserved are shown within boxes. The sequence number shown on the top of the figure is based on the sequence of human MSP.

$\mathrm{OD}_{600}$ reached 0.6 , and the culture was then incubated for another $4 \mathrm{~h}$. After harvesting the $E$. coli by centrifugation at $2000 \times g$ for $20 \mathrm{~min}$ at $4^{\circ} \mathrm{C}$, the pellet was washed with $40 \mathrm{ml}$ resuspension buffer $(50 \mathrm{mM}$ sodium phosphate, $200 \mathrm{mM}$ sodium chloride, $\mathrm{pH} 8.0$ ). The bacteria were then disrupted by a microfluidizer and the supernatant was recovered by centrifugation at $50000 \times g$ for $30 \mathrm{~min}$ at $4^{\circ} \mathrm{C}$. The supernatant was mixed with $6 \mathrm{ml}$ nickel-nitrilotriacetic acid (Ni-NTA) resin for $1 \sim 2 \mathrm{~h}$, and then loaded in a $40 \mathrm{ml}(25 \times 100 \mathrm{~mm})$ column. The column was first washed with the sufficient washing buffer $(5 \mathrm{mM}$ imidazole in $50 \mathrm{mM}$ sodium phosphate, $200 \mathrm{mM}$ sodium chloride, $\mathrm{pH} 8.0$ ) to remove the contaminants. The binding protein was finally eluted with washing buffer containing $120 \mathrm{mM}$ imidazole. To improve the purity of recombinant protein, the reversed-phase high performance liquid chromatography (HPLC) was applied for further purification. The purity of the recombinant porcine MSP was checked by sodium dodecyl sulfate-polyacrylamide gel electrophoresis (SDSPAGE) and emission/mass spectrometry.

\subsection{Circular dichroism (CD) experiment}

CD experiments were carried out using an Aviv 202 SF CD spectrometer (Lakewood, NJ, USA) calibrated with (+)-10-camphorsulfonic acid (CSA) at $25^{\circ} \mathrm{C}$. In general, a $2 \mathrm{~mm}$ path-length cuvette with $20 \mu \mathrm{M}$ porcine MSP in $20 \mathrm{mM}$ phosphate was used for $\mathrm{CD}$ experiments. The steady-state CD spectra were recorded from 180 to $260 \mathrm{~nm}$ at different temperatures and $\mathrm{pH}$ values. After background subtraction and smoothing, all the $\mathrm{CD}$ data were converted from $\mathrm{CD}$ signal (millidegree) into mean residue ellipticity $\left(\mathrm{deg} \mathrm{cm}^{2} \mathrm{dmol}^{-1}\right)$. The secondary structure content was estimated from the CD spectra according to the methods of CONTIN, SELCON and CDSSTR [12]. Equilibrium thermal denaturing experiments were performed by measuring the change of ellipticity at $231 \mathrm{~nm}$.

\subsection{NMR experiment}

All NMR experiments were performed on a Bruker AVANCE 600 spectrometer equipped with a triple $\left({ }^{1} \mathrm{H},{ }^{13} \mathrm{C}\right.$ and $\left.{ }^{15} \mathrm{~N}\right)$ resonance probe including a shielded $\mathrm{z}$-gradient. NMR samples were prepared in $50 \mathrm{mM}$ phosphate buffer in $90 \% \mathrm{H}_{2} \mathrm{O} / 10 \% \mathrm{D}_{2} \mathrm{O}$ at $\mathrm{pH} 5.0$ and contained $0.35 \mathrm{ml}$ of $1.5 \mathrm{mM}$ protein in a Shigemi NMR tube (Allison Park, PA, USA). For the native porcine MSP, two-dimensional (2D) ${ }^{1}$ H NMR spectra, scalar-correlated spectroscopy (COSY) [13], total correlation spectroscopy (TOCSY) [14] and nuclear Overhauser enhancement spectroscopy (NOESY) [15] spectra were collected. All heteronuclear NMR experiments for recombinant MSP were carried out as described elsewhere [16]. The amide proton exchange rates were identified from residual amide proton signals observed in several 2D TOCSY and ${ }^{1} \mathrm{H}^{15} \mathrm{~N}$ heteronuclear single-quantum correlation (HSQC) spectra recorded at $310 \mathrm{~K}$ and $\mathrm{pH} 5.0$ in $\mathrm{D}_{2} \mathrm{O}$. 2,2-Dimethyl-2-silapentane-5-sulfonate (DSS) was used as an external chemical shift standard at $0.00 \mathrm{ppm}$. The ${ }^{15} \mathrm{~N}$ and ${ }^{13} \mathrm{C}$ chemical shifts were indirectly referenced using the consensus $\Xi$ ratios of the zero-point frequencies at $310 \mathrm{~K}$ [17]. All spectra were processed using XWINNMR and analyzed using AURELIA on an SGI $\mathrm{O}_{2}$ workstation. Linear prediction was applied in the ${ }^{13} \mathrm{C}$ and ${ }^{15} \mathrm{~N}$ dimensions to improve the digital resolution.

\subsection{Tertiary structure calculations}

Distance restraints of porcine MSP were derived primarily from three-dimensional (3D) NOESY-HSQC spectra recorded in aqueous solution at $310 \mathrm{~K}, \mathrm{pH}$ 5.0. Peak intensities were classified as large, medium, small, and very small, corresponding to upper bound interproton distance restraints of $2.5,3.5,4.5$, and $6.0 \AA$, respectively. An additional correction of $1.0 \AA$ was added for methylene and methyl groups. The backbone $\phi, \psi$ torsion angles were calculated using the TALOS program [18] and those in good agreement with nuclear Overhauser enhancement (NOE) correlations were used for structure generations. All minimization and dynamical simulated annealing calculations were carried out with the program X-PLOR 98 [19] on an SGI $\mathrm{O}_{2}$ workstation. The INSIGHT II (Molecular Simulation Inc., San Diego, CA, USA), MOLMOL [20] and GRASP [21] programs were used to visually observe sets of structures and to calculate and generate the electrostatic surface potential of the final 3D models. The distributions of the backbone dihedral angles of the final converged structures were evaluated using PROCHECK-NMR [22]

\section{Results}

\subsection{Conformational stability and secondary structure based on CD data}

CD spectra of the recombinant porcine MSP at different $\mathrm{pH}$ values are very similar, indicating that its secondary structure is independent of $\mathrm{pH}$ factors (Fig. 2A). Also, CD spectra of the native and recombinant proteins acquired under identical conditions are similar (Fig. 2B), revealing that they possess very similar secondary structures. The secondary structure contents of the recombinant and native porcine MSPs estimated using CONTIN-LL, SELCON3 and CDSSTR programs [12] showed that porcine MSP is mainly composed of $\beta$-sheet structures and contains a very small content of $\alpha$-helix, if any. Since both native and recombinant proteins are not completely denatured even at temperature as high as $95^{\circ} \mathrm{C}$, their $T_{\mathrm{m}}$ values could not be accurately obtained, but we could conclude that both proteins are highly thermal stable with an almost identical $T_{\mathrm{m}}$ value since the profile of their titration curves could be nicely superimposed. 


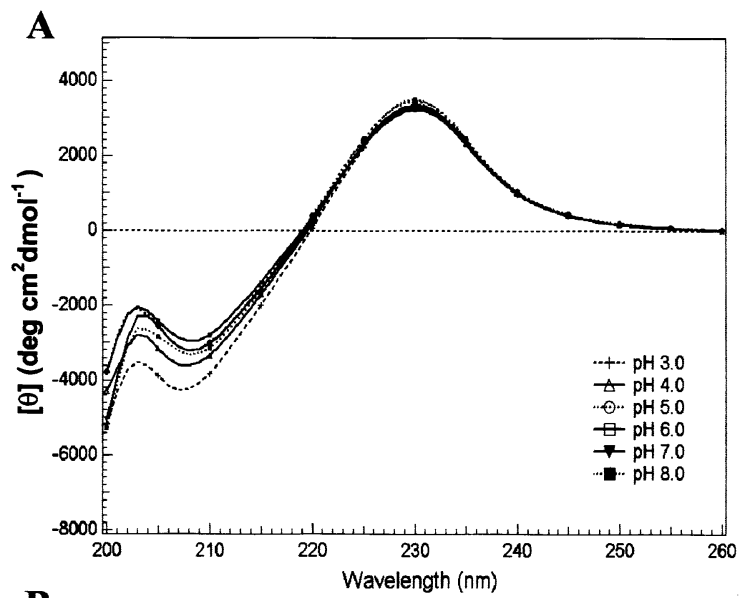

B

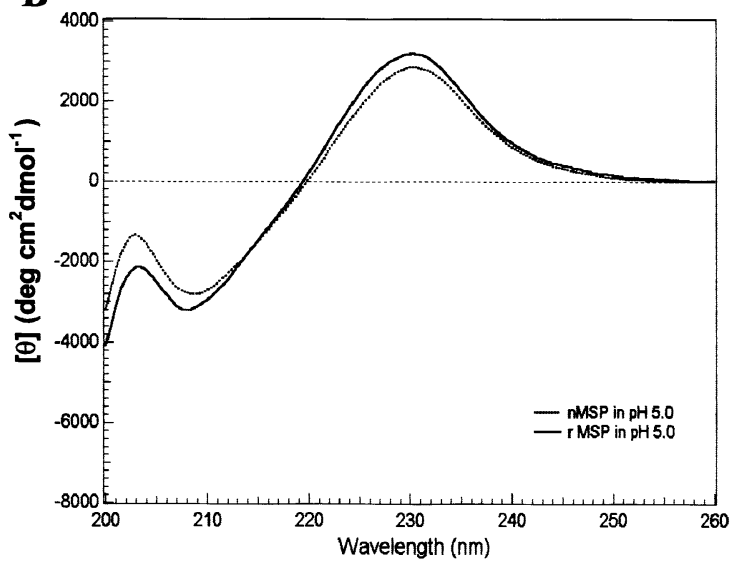

Fig. 2. A: CD spectra of the recombinant porcine MSP at different $\mathrm{pH}$ values are similar, revealing that the secondary structure of porcine MSP is $\mathrm{pH}$ independent at $\mathrm{pH}$ 3.0-8.0. B: CD spectra between native and recombinant porcine MSPs under the identical conditions are very much alike, indicating that their secondary structures are similar.

\subsection{Comparison of $N M R$ data and NMR assignment}

Fig. 3A shows a comparison of one-dimensional (1D) spectra between native and recombinant porcine MSPs. It was found that most of the residues display identical chemical shifts, indicating that their tertiary structures are also very much alike. In addition, we checked and compared NOE connectivities, particularly long-range NOEs, between native and recombinant proteins. Again, we did not observe any significant change and this finding is in good agreement with the result based on the change of chemical shifts.

With high stability and well-dispersed NMR data, porcine MSP is suitable for NMR structural studies. We chose pH 5.0 for the structural study since under this condition the sample possesses slow amide exchange in addition to having well-resolved NMR data. Sequence-specific assignment of the backbone atoms was achieved by independent connectivity analysis of $\mathrm{CBCA}(\mathrm{CO}) \mathrm{NH}, \mathrm{HNCACB}, \mathrm{HNCO}, \mathrm{HN}(\mathrm{CA}) \mathrm{CO}$ and $\mathrm{C}(\mathrm{CO}) \mathrm{NH}$. The ${ }^{1} \mathrm{H}$ resonances were assigned using 3D TOCSY-HSQC, HAHB(CO)NH, HCCH-TOCSY and $\mathrm{HC}(\mathrm{CO}) \mathrm{NH}$. Combined information from $2 \mathrm{D}{ }^{1} \mathrm{H}-{ }^{15} \mathrm{~N}$ HSQC and 3D NOESY-HSQC experiments yielded assignments for the side-chain amide resonances of the Asn and Gln residues. Aromatic resonances were assigned using 2D
${ }^{1} \mathrm{H}-{ }^{13} \mathrm{C}$ HSQC, 2D NOESY and 2D TOCSY data in $\mathrm{D}_{2} \mathrm{O}$. All proline residues (Pro ${ }^{6}, \mathrm{Pro}^{12}, \mathrm{Pro}^{24}, \mathrm{Pro}^{53}$ and $\mathrm{Pro}^{80}$ ) were assigned by sequential connectivities. We have so far assigned the resonances of all backbone ${ }^{15} \mathrm{~N},{ }^{1} \mathrm{H}^{\mathrm{N}}, \mathrm{H}^{\alpha},{ }^{13} \mathrm{C}^{\alpha},{ }^{13} \mathrm{C}^{\prime}$ and side-chain ${ }^{13} \mathrm{C}^{\beta}$, with the exception of ${ }^{1} \mathrm{H}^{\mathrm{N}}$ resonance in Lys ${ }^{32}$, which is presumably exposed to the surface and has a very fast exchange rate with $\mathrm{H}_{2} \mathrm{O}$. The backbone ${ }^{1} \mathrm{H},{ }^{13} \mathrm{C}$ and ${ }^{15} \mathrm{~N}$ chemical shifts of porcine MSP at $\mathrm{pH} 5.0$ and $310 \mathrm{~K}$ have been deposited to BioMagResBank under accession number BMRB-5565. Based on the CD and NMR data comparison, we concluded that the extra 17 residues at the $\mathrm{N}$-terminus in the recombinant protein do not affect the conformation of the mature protein. Thus, we did not attempt to assign resonances of these residues. The resonance assignments are clearly annotated in the $2 \mathrm{D}{ }^{1} \mathrm{H}_{-}{ }^{15} \mathrm{~N}$ HSQC spectrum (Fig. 3B).

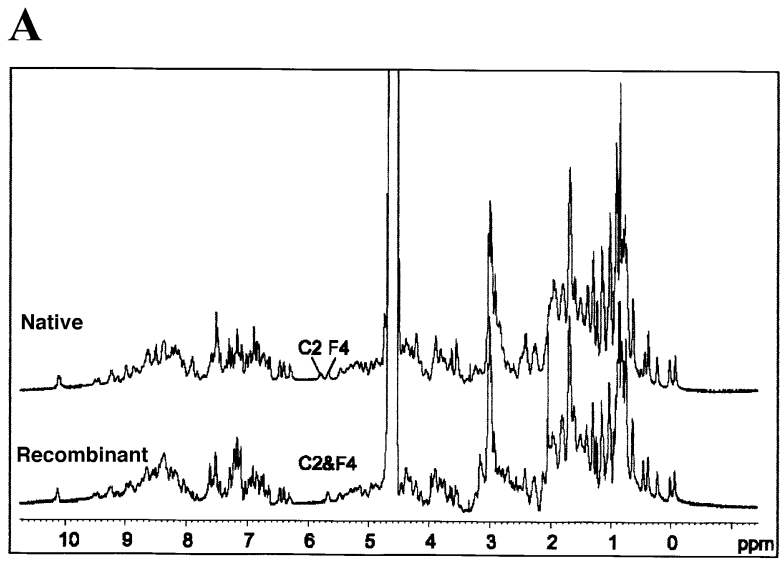

B

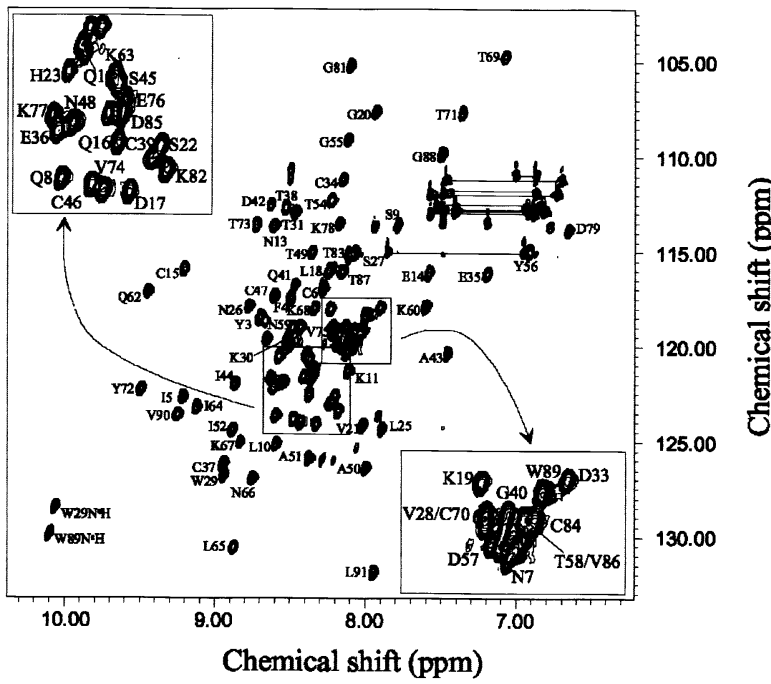

Fig. 3. A: Comparison of 1D spectra of the native and recombinant porcine MSPs is shown. Due to the effect of the extra 17 residues at the $\mathrm{N}$-terminus in the recombinant protein, a few small regions of chemical shifts are different. B: A $2 \mathrm{D}{ }^{1} \mathrm{H}^{-15} \mathrm{~N}$ HSQC spectrum of recombinant porcine MSP obtained at $310 \mathrm{~K}$. The NMR sample contained about $1.5 \mathrm{mM}$ porcine MSP in $50 \mathrm{mM}$ phosphate buffer, $\mathrm{pH}$ 5.0. The resonance assignments are indicated with the one-letter amino acid code and residue number. Side-chain amide protons of Asn and Gln are indicated by horizontal lines. 
$\mathbf{A}$

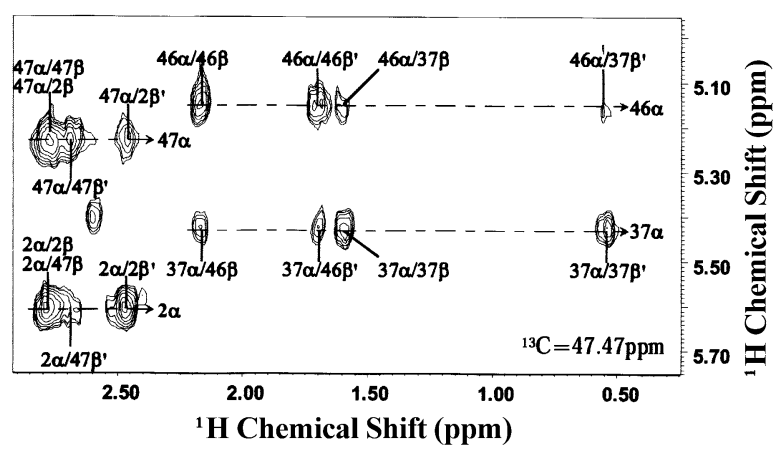

B

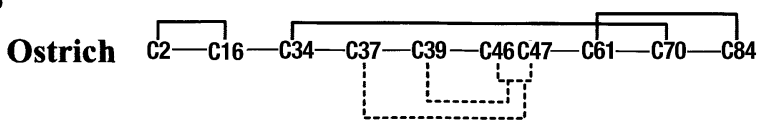

Porcine

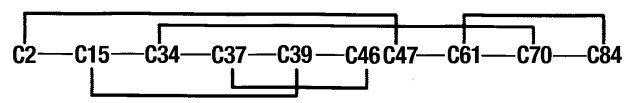

Fig. 4. A: A partial region of $2 \mathrm{D}$ NOESY spectrum extracted from $3 \mathrm{D}{ }^{13} \mathrm{C}$-NOESY-HSQC in $\mathrm{D}_{2} \mathrm{O}$ at ${ }^{13} \mathrm{C}=47.47 \mathrm{ppm}$ shows the NOE connectivities of two disulfide pairings, $\mathrm{C} 2-\mathrm{C} 47$ and $\mathrm{C} 37-\mathrm{C} 46$. $\mathrm{B}$ : Comparison of the disulfide pairings between ostrich and porcine MSPs is shown. The disulfide pairings for ostrich MSP are determined based on MALDI-TOF mass analysis [8] and the two disulfide pairings that could not be unambiguously identified are indicated by a dotted line.

\subsection{Disulfide pairings and proline cis-trans conformation}

Based on the detection of $d_{\beta \beta}(i, j)$ and/or $d_{\alpha \beta}(i, j)$ NOEs (Fig. 4A) between the two Cys residues that form a disulfide bridge, the connections of five disulfide bridges in porcine MSP, which include $\mathrm{Cys}^{2}-\mathrm{Cys}^{47}, \mathrm{Cys}^{15}-\mathrm{Cys}^{39}, \mathrm{Cys}^{34}-\mathrm{Cys}^{70}, \mathrm{Cys}^{37}$ $\mathrm{Cys}^{46}$, and $\mathrm{Cys}^{61}-\mathrm{Cys}^{84}$, were unambiguously assigned. Interestingly, these pairings are different from the pairings of ostrich MSP derived based on MALDI-TOF techniques [8]. In comparison only two pairings (34-70 and 61-84) are the same among the five disulfide pairings between porcine and ostrich MSPs (Fig. 4B). There are five proline residues in the sequence and they all form trans conformations, based on the criteria that all prolines possess strong $d_{\alpha \delta}(i, i+1)$ NOEs with their preceding residues.

\subsection{Secondary structure and amide proton exchange rate}

A consensus chemical shift index (CSI) [23] generated using ${ }^{1} \mathrm{H}^{\alpha},{ }^{13} \mathrm{C}^{\alpha},{ }^{13} \mathrm{C}^{\beta}$, and ${ }^{13} \mathrm{C}^{\prime}$ chemical shifts revealed that the secondary structure of porcine MSP is primarily composed of eight $\beta$-strands (data not shown) and contains no $\alpha$-helical structure. In addition, we did not detect characteristic $\alpha$-helical NOEs, $d_{\alpha \mathrm{N}}(i, i+3), d_{\alpha \beta}(i, i+3)$, and $d_{\mathrm{NN}}(i, i+1)$, in the NOESY data, further indicating the absence of $\alpha$-helix for porcine MSP. Given the observed crossover NOEs between the $\beta$-strands and the slowly exchanging amide protons (Fig. 5A), we concluded that these $\beta$-strands clearly form a fourstranded $\left(\mathrm{Cys}^{2}-\mathrm{Ile}^{5}, \mathrm{Ser}^{27}-\mathrm{Thr}^{31}, \mathrm{Glu}^{36}-\mathrm{Cys}^{39}\right.$ and $\mathrm{Ile}^{44}$ $\left.\mathrm{Asn}^{48}\right)$ and two double-stranded antiparallel $\beta$-sheets $\left(\mathrm{Cys}^{61}\right.$ $\mathrm{Thr}^{69}$ and $\mathrm{Thr}^{71}-\mathrm{Lys}^{77}$; $\mathrm{Ile}^{52}-\mathrm{Thr}^{54}$ and $\mathrm{Gly}^{88}-\mathrm{Leu}^{91}$ ), as represented by the topology diagram of Fig. 5B. A detailed comparison of exchange rate data reveals that all of very slowly amide protons $\left(\mathrm{Ile}^{5}, \mathrm{Trp}^{29}, \mathrm{Glu}^{36}, \mathrm{Thr}^{38}\right.$, and $\mathrm{Ser}^{45}$ ) are located at the four-stranded antiparallel $\beta$-sheet, indicating that this $\beta$-sheet region possesses stronger $\mathrm{H}$ bonds and is the most stable in the structure of porcine MSP. In comparison, there is no medium or slow amide proton observed in the doublestranded $\beta$-sheet $\left(\mathrm{Ile}^{52}-\mathrm{Thr}^{54}\right.$ and $\mathrm{Gly}^{88}-\mathrm{Leu}^{91}$ ), revealing that this $\beta$-sheet is the least stable among all $\beta$-sheet structures.

\subsection{Structure calculation}

To further confirm the disulfide pairings of porcine MSP using structural calculations, we initially built three starting structures of porcine MSP with different disulfide pairings, that is, a structure with our derived disulfide pairings ( $\mathrm{P} 1$ : 2-47, 15-39, 34-70, 37-46 and 61-84) and two structures based on disulfide pairings of ostrich MSP (P2: 2-15, 34 $70,37-46,39-47$ and $61-84 ;$ P3: 2-15, 34-70, 37-47, 39-46 and 61-84). A set of 90 restraints, including 43 long-range distance restraints, 32 dihedral angle restraints, and 15 hydrogen bond restraints, was applied for simulated annealing and energy minimization calculations using the program X-PLOR. 30 structures were generated for each of P1, P2 and P3, and
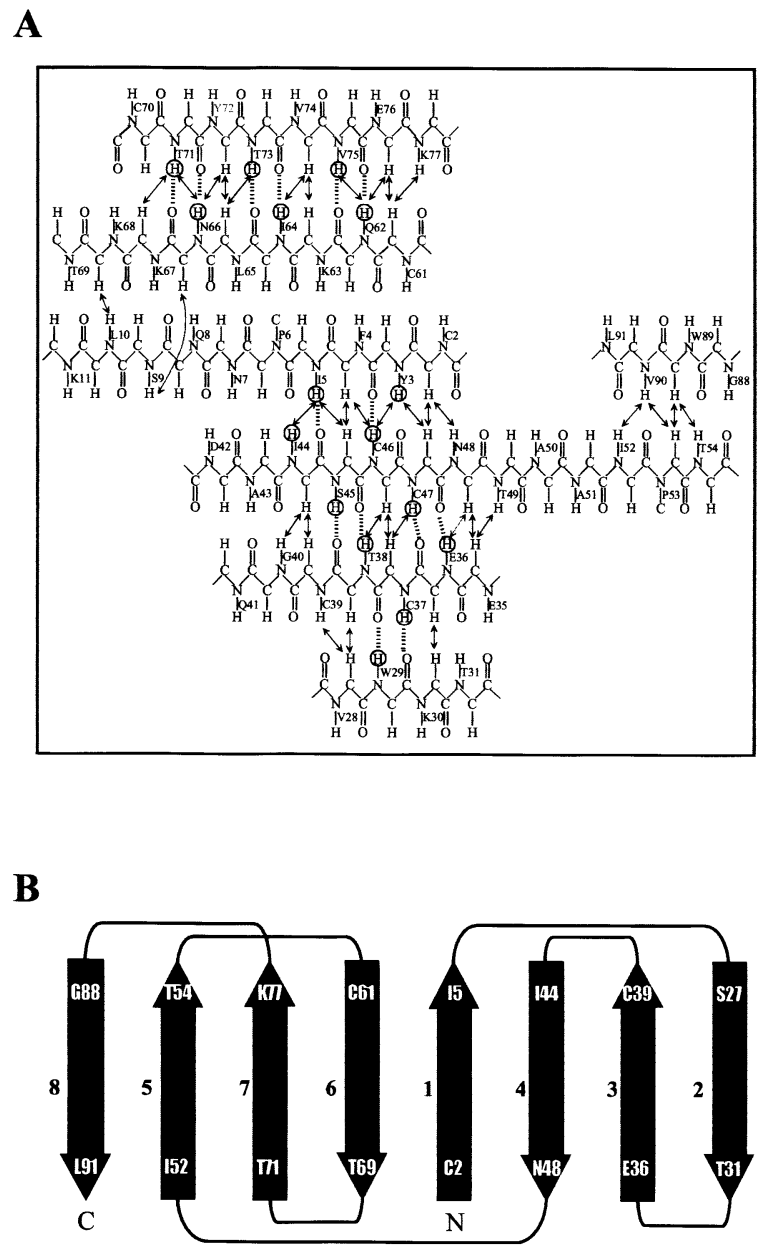

Fig. 5. A: Definition of the $\beta$-sheet structure of porcine MSP is shown based on the NOEs and amide proton exchange rate. Crossover NOEs between $\beta$-strands are indicated by double arrows. Dashed lines between backbone amide protons and backbone carbonyl oxygens indicate hydrogen bonds consistent with slow exchanging $\mathrm{H}^{\mathrm{N}}$ observed in $\mathrm{D}_{2} \mathrm{O}$. The amide protons with slow exchange rates are circled. $\mathrm{B}$ : The topology diagram of porcine MSP is shown. For clarity, the residues that form a $\beta$-strand are specified. 
Table 1

The average energy $(\mathrm{kcal} / \mathrm{mol})$ of each target function from the X-PLOR program for the P1, P2, and P3 final structures, which were generated using three different disulfide pairings (P1: 2-47, 15-39, 34-70, 37-46 and 61-84; P2: 2-15, 34-70, 37-46, 39-47 and 61-84; P3: 2-15, 34-70, $37-47,39-46$ and $61-84)$

\begin{tabular}{llllllrr}
\hline Structure type & $E$ (total) & $E$ (bond) & $E$ (angle) & $E$ (impr) & $E$ (VDW) & $E(\mathrm{CDIH})$ & $E$ (NOE) \\
\hline P1 & $396.2 \pm 7.9$ & $36.1 \pm 0.9$ & $208.9 \pm 4.8$ & $14.2 \pm 0.6$ & $131.5 \pm 4.8$ & $3.9 \pm 0.7$ & $1.5 \pm 0.8$ \\
P2 & $514.0 \pm 16.4$ & $44.1 \pm 2.5$ & $245.3 \pm 1.3$ & $25.0 \pm 2.8$ & $160.3 \pm 7.7$ & $13.2 \pm 1.7$ & $26.2 \pm 9.9$ \\
P3 & $444.6 \pm 19.5$ & $36.4 \pm 1.5$ & $215.4 \pm 8.0$ & $18.1 \pm 3.3$ & $138.1 \pm 3.9$ & $4.5 \pm 0.9$ & $32.0 \pm 6.9$ \\
\hline
\end{tabular}

the best five structures of each type were chosen for analysis. Table $1>0.5 \AA$ for distance restraints of $d_{\alpha \alpha}(35,48)$ and $d_{\alpha \alpha}>1.0 \AA$ for distance restraint of $d_{\alpha \alpha}(37,46)$. In addition, structural analysis using PROCHECK-NMR also revealed that P1 structure is the best, due to the higher percentage of its residues which lie in the most favored and additional allowed regions (plot not shown). As a result, the structural calculation further proves the accuracy of disulfide pairings determined using NOE correlations for porcine MSP.

\section{Discussion}

The similarities of CD and NMR spectra between native and recombinant proteins indicate the correct tertiary fold for the recombinant porcine MSP. Based on the specific NOEs, we identified the five disulfide pairings of porcine MSP. To ensure the accuracy of these disulfide pairings, we further applied structural calculations using 90 restraints, which were all double checked and included several key long-range distances in $\beta$-sheet regions. Of three types of structures generated, only P1 structures that were generated using our disulfide pairings could fit these restraints. Accordingly, we believe that our disulfide pattern assignments for porcine MSP are correct. However, we still don't know why porcine and ostrich MSP exhibit different disulfide pairings. One possible explanation is that due to the rapid evolution and low sequence identities, MSPs intrinsically possess different disulfide patterns even though Cys residues are all conserved and located at the same positions. Obviously, to further confirm the existence of different disulfide patterns on MSPs, more indepth structural study, especially in the identification of disulfide patterns, is needed. Furthermore, the secondary structure of porcine MSP was determined to be composed of a fourstranded and two double-stranded antiparallel $\beta$-sheets. As we know, this is the first detailed secondary structure reported among several types of MSPs. The disulfide patterns and the secondary structure on porcine MSP determined in this work are novel and can provide valuable information for subsequent MSP structural studies and additional data for understanding the biological functions of MSP.

Acknowledgements: We would like to thank Academia Sinica and the National Science Council, Taiwan, ROC for support of this work. The NMR spectra were obtained at the High-field Biomacromolecular NMR Core Facility at Academia Sinica, supported by the National Science and Technology Program for Medical Genomics.

\section{References}

[1] Lilja, H. and Abrahamsson, P.A. (1988) Prostate 12, 29-38.

[2] Makinen, M., Valtonen-Andre, C. and Lundwall, A. (1999) Eur. J. Biochem. 264, 407-414.

[3] Xuan, J.W., Wu, D., Guo, Y., Garde, S., Shum, D.T., Mbikay, M., Zhong, R. and Chin, J.L. (1997) DNA Cell Biol. 16, 627638.

[4] Nolet, S., St-Louis, D., Mbikay, M. and Chretien, M. (1991) Genomics 9, 775-777.

[5] Fernlund, P., Granberg, L.B. and Roepstorff, P. (1994) Arch. Biochem. Biophys. 309, 70-76.

[6] Fernlund, P., Granberg, L.B. and Larsson, I. (1996) Arch. Biochem. Biophys. 334, 73-82.

[7] Xuan, J.W., Kwong, J., Chan, F.L., Ricci, M., Imasato, Y., Sakai, H., Fong, G.H., Panchal, C. and Chin, J.L. (1999) DNA Cell Biol. 18, 11-26.

[8] Lazure, C., Villemure, M., Gauthier, D., Naude, R.J. and Mbikay, M. (2001) Protein Sci. 10, 2207-2218.

[9] Kamada, M., Mori, H., Maeda, N., Yamamoto, S., Kunimi, K., Takikawa, M., Maegawa, M., Aono, T., Futaki, S. and Koide, S.S. (1998) Biochim. Biophys. Acta 1388, 101-110.

[10] Weiber, H., Borch, K., Sundler, F. and Fernlund, P. (1999) Digestion $60,440-448$

[11] Chao, C.F., Chiou, S.T., Jeng, H. and Chang, W.C. (1996) Biochem. Biophys. Res. Commun. 218, 623-628.

[12] Sreerama, N. and Woody, R.W. (2000) Anal. Biochem. 287, 252 260.

[13] Rance, M., Sorensen, O.W., Bodenhausen, G., Wagner, G., Ernst, R.R. and Wuthrich, K. (1983) Biochem. Biophys. Res. Commun. 117, 479-485.

[14] Bax, A. and Davis, D.G. (1985) J. Magn. Reson. 65, 355-360.

[15] Kumar, A., Ernst, R.R. and Wuthrich, K. (1980) Biochem. Biophys. Res. Commun. 95, 1-6.

[16] Kay, L.E. (1995) Prog. Biophys. Mol. Biol. 63, 277-299.

[17] Sykes, B.D., Wishart, D.S., Bigam, C.G., Yao, J., Abildgaard, F., Dyson, H.J., Oldfield, E. and Markley, J. (1995) J. Biomol. NMR 6, 135-140.

[18] Cornilescu, G., Delaglio, F. and Bax, A. (1999) J. Biomol. NMR $13,289-302$.

[19] Brunger, A.T. (1998) X-PLOR version 98, Yale University Press, New Haven, CT.

[20] Koradi, R., Billeter, M. and Wuthrich, K. (1996) J. Mol. Graph. $14,29-32$.

[21] Nicholls, A., Sharp, K.A. and Honig, B. (1991) Proteins 11, 281296.

[22] Laskowski, R.A., Rullmannn, J.A., MacArthur, M.W., Kaptein, R. and Thornton, J.M. (1996) J. Biomol. NMR 8, 477-486.

[23] Sykes, B.D. and Wishart, D.S. (1994) Methods Enzymol. 239, 363-392.

[24] Higgins, D.G., Thompson, J.D. and Gibson, T.J. (1996) Methods Enzymol. 266, 383-402.

[25] Gouet, P., Courcelle, E., Stuart, D.I. and Metoz, F. (1999) Bioinformatics $15,305-308$. 